\title{
Primer registro para el sur del Perú de Rhynchoconger nitens (Jordan \& Bollman 1890) (Teleostei: Congridae). ¿Un caso de dispersión larvaria relacionada con El Niño?
}

\author{
First record of Rhynchoconger nitens (Jordan \& Bollman 1890) (Teleostei: Congridae) from off \\ southern Peru. A case of larval dispersal in relation with El Niño? \\ Philippe Béarez*, Hugo Treviño** y Martín Zambrano** \\ *Instituto Francés de Estudios Andinos (IFEA), Contralmirante Montero 141, Casilla 18-1217, Lima 18 \\ **Instituto del Mar del Perú (IMARPE), Laboratorio costero de Ilo \\ bearez@ifeanet.org
}

\begin{abstract}
Resumen.- Se registra por primera vez en el sur del Perú (Ilo, Moquegua) un ejemplar de Rhynchoconger nitens, pequeño congrio de la familia Congridae. Para explicar su presencia en $18^{\circ}$ de latitud sur, se propone una hipótesis de migración larvaria durante un fenómeno de El Niño.

Palabras claves: biogeografía, dispersión larvaria, El Niño, Perú, Rhynchoconger nitens.
\end{abstract}

\begin{abstract}
A specimen of Rhyncoconger nitens, a small congrid, is recorded for the first time from Ilo (Moquegua, Peru). To explain its presence at $18^{\circ}$ of south latitude, a hypothesis of larval migration during an El Niño is proposed. Keywords: biogeography, larval dispersal, El Niño, Peru, Rhynchoconger nitens.
\end{abstract}

\section{Introducción}

La Familia Congridae cuenta con cerca de ciento cincuenta especies repartidas sobre todo el planeta en aguas tropicales y templadas (Nelson 1994). Está representada en el Pacífico Tropical Este por una decena de géneros y unas quince especies (Smith 1995) y en las aguas peruanas por cuatro especies (Chirichigno \& Vélez 1998):

- Paraconger californiensis Kanazawa 1961;

- Xenomystax atrarius Gilbert 1891;

- Gnathophis cinctus (Garman 1899);

- Bathycongrus varidens (Garman 1899).

En cuanto al género Rhynchoconger, consiste en un grupo pequeño de cinco especies de congrios de los cuales uno sólo vive en el Pacífico Este: Rhynchoconger nitens (Jordan \& Bollman 1890). Chirichigno \& Cornejo (2001) por primera vez lo señalan para la mitad norte del Perú, hasta Huarmey $\left(10^{\circ} \mathrm{S}\right)$, pero sin mas datos.

El hecho de encontrar por primera vez este congrio en aguas sureñas del Perú y de que no haya sido registrado en forma objetiva hasta ahora, nos hace pensar que no es un habitante usual del litoral sur peruano, sino que más bien se trataría de una intrusión casual.

\section{Materiales y Métodos}

El ejemplar fue entregado al laboratorio costero del Instituto del Mar del Perú (IMARPE) por un pescador del terminal pesquero de Ilo (Dpto. de Moquegua): con fecha 17 de junio de 1998. Según su comentario, fue capturado con cordel cerca de la orilla, en las inmediaciones del puerto. El congrio se conserva actualmente en la colección del Muséum national d'histoire naturelle de París (Francia) con el número MNHN 2001-1321.

También se observaron tres otros ejemplares, recolectados por el autor en Ecuador y Panamá y conservados en el MNHN con los números 1997-3821, 2001-1279 y 2001-1372.

Las medidas se registraron según la metodología de Böhlke (1989) y Smith (1989). Se expresan en milímetros y en porcentajes de la longitud preanal; se escogió esa medida por el alto porcentaje de colas regeneradas, lo que le da mejor representatividad que la longitud total. Las abreviaturas empleadas son las siguientes: DIO: distancia interorbitaria, DO: diámetro cránio-caudal del ojo, LC: longitud de la cabeza, LH: longitud del hocico, LMS: longitud de la maxilla superior, LP: longitud de la aleta pectoral, LPA: longitud preanal, LPD: longitud predorsal, LT: longitud total. 
El holotipo conservado en el National Museum of Natural History de Washington (Estados Unidos) con el número USNM 44395 no fue examinado, sus medidas se reconstituyeron sobre la base de la descripción original. El número total de vértebras fue comunicado por D. Smith.

\section{Resultados y Discusión}

\section{Familia Congridae}

Subfamilia Congrinae

Rhynchoconger nitens (Jordan \& Bollman 1890)

Ophisoma nitens Jordan \& Bollman 1890: 153. Proc. U. S. Natl. Mus. 12(770). Frente a la costa pacífica de Panamá/Colombia, 0847'00"N, 79²9'30"W, estación 2801, profundidad: $26 \mathrm{~m}$. Holotipo (único): USNM 44395.
Hildebrandia Jordan \& Evermann 1927 se usa por primera vez para $R$. nitens por Raju (1985). Según Smith (1989), es un género sinónimo y, aunque a menudo usado en la literatura, Hildebrandia nitens es una nueva combinación no válida.

Ariosoma Swainson 1838 es un género de la subfamilia Bathymyrinae (Smith 1995), por lo tanto tan poco se puede utilizar la combinación Ariosoma nitens, tal como se ve en Massay (1983) y Chirichigno \& Cornejo (2001).

Material examinado: un ejemplar juvenil de $193 \mathrm{~mm}$

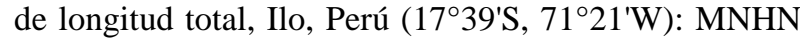
2001-1321 (Fig. 1). Un ejemplar adulto 308 mm LT, Panamá (845’N, 7906’W): MNHN 2001-1279. Dos ejemplares adultos provenientes de Puerto López, Ecuador (1³3’S, 8049’W): 345 mm LT, MNHN 20011372 y 463 mm LT, MNHN 1997-3821.

Morfometría: ver Tabla 1.

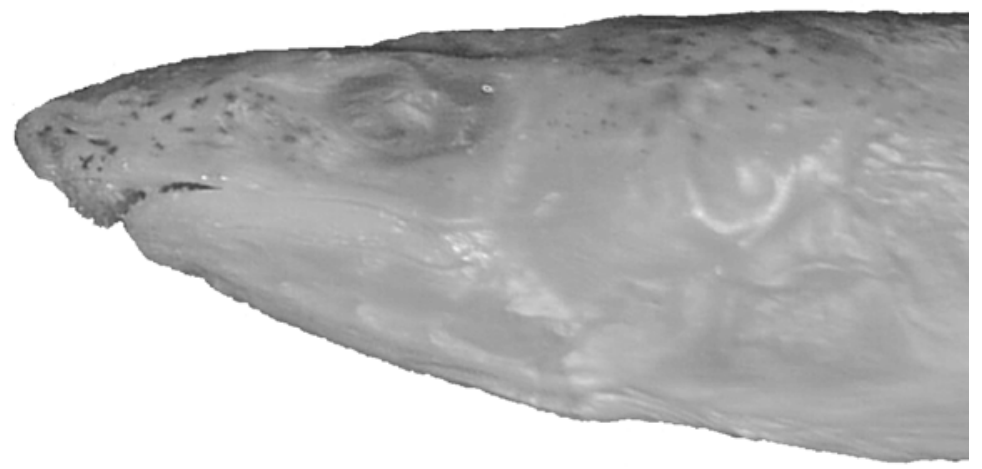

Figura 1

Rhynchoconger nitens, especimen de 193 mm LT de Perú. (Foto P. Béarez)

Rhynchoconger nitens, 193 mm LT specimen from Peru. (Photo P. Béarez)

Tabla 1

Datos morfométricos de los 4 especímenes examinados y comparación con el holotipo (datos reconstituidos a partir de la descripción original). (*: cola regenerada)

Morfometric data of the 4 studied specimens and comparison with the holotype (data reconstituted from the original description). (*: regenerated tail)

\begin{tabular}{|l|c|c|c|c|}
\hline & $\begin{array}{c}\text { Perú } \\
\text { MNHN 2001-1321 }\end{array}$ & $\begin{array}{c}\text { Ecuador } \\
\text { MNHN 2001-1372 - } \\
\text { MNHN 1997-3821 }\end{array}$ & $\begin{array}{c}\text { Panamá } \\
\text { MNHN 2001-1279 }\end{array}$ & $\begin{array}{c}\text { Colombia } \\
\text { Holotipo } \\
\text { USNM 44395 }\end{array}$ \\
\hline LT (mm) & 193 & $345-463$ & 308 & 228,6 \\
\hline LPA (mm) & 55,5 & $104-154$ & 107,5 & 62,4 \\
\hline LPD (\%LPA) & 46,8 & $47,1-46,1$ & 46,5 & - \\
\hline LC (\%LPA) & 48,6 & $46,3-46,8$ & 47,4 & 48,2 \\
\hline LH (\%LPA) & 11,7 & $11,7-11,0$ & 11,6 & - \\
\hline LMS (\%LPA) & 20,7 & $18,5-18,2$ & 18,6 & 17,1 \\
\hline LP (\%LPA) & 15,5 & $15,0-14,9$ & 15,1 & 6,9 \\
\hline DO (\%LPA) & 6,5 & $6,3-6,0$ & 6,5 & 3,4 \\
\hline DIO (\%LPA) & 4,9 & $5,5-5,4$ & 5,2 & $152^{*}$ \\
\hline Total vértebras & 165 & $179-132 *$ & $130 *$ & \\
\hline
\end{tabular}


Distribución: $R$. nitens es una especie endémica del Pacífico Tropical Este y su ámbito de distribución abarca desde el Golfo de California hasta Ecuador (Smith 1995, Béarez 1996). No se conocen registros de las Islas Galápagos. En el presente trabajo se extiende considerablemente el límite sur de su distribución, hasta el extremo sur de Perú. También se encuentran en el USNM dos especímenes capturados en la mitad norte del Perú : USNM 208319, 29/09/1971, 45-46 m (3¹7'S, $\left.80^{\circ} 52^{\prime} \mathrm{W}\right)$ y USNM 208320, 3/12/1971, $85 \mathrm{~m}$ (5²3'S, $81^{\circ} 19^{\prime} \mathrm{W}$ to $\left.5^{\circ} 38^{\prime} \mathrm{S}, 8^{\circ} 18^{\prime} \mathrm{W}\right)$. Cabe mencionar la presencia en la Scripps Institution of Oceanography de una larva (SIO 69-497) capturada el 9/12/1969 en 675 m de profundidad en pleno océano ( $\left.8^{\circ} 38^{\prime} \mathrm{S}, 103^{\circ} 46 \mathrm{~W}\right)$, lo que demuestra la amplia distribución de las larvas de la especie en el Pacífico tropical este.

Se trata de una especie pequeña que generalmente no pasa de unos $40 \mathrm{~cm}$ de longitud total; el ejemplar de 463 mm LT, proveniente de Ecuador, es el más grande conocido (peso en fresco : 105 g). Rhynchoconger nitens se caracteriza por una cola alargada y aguzada, bordeada de negro, un hocico bien prolongado delante de la maxilla inferior y dientes intermaxillares en placa totalmente expuesta cuando la boca se encuentra cerrada. El número total de vertebras oscila entre 168 y 182 (Raju 1985).

Los congrios adultos son generalmente bentónicos y viven enterrados o metidos en huecos del sustrato. También son generalmente de hábitos nocturnos, como lo es $R$. nitens (obs. pers.). Como todos los elopomorfos, tienen larvas peculiares en forma de hojita alargada o cinta, denominada leptocéfala. Esas larvas son pelágicas y forman buena parte del ictioplancton; dentro de los congrios del Pacífico Este la larva de $R$. nitens es una de las más abundantes (Raju 1985).

Creemos que se puede explicar la presencia de un individuo juvenil de esta especie en el extremo sur de Perú, a unos 2000 km al sur de su distribución natural, por una migración de larvas con las corrientes cálidas durante el evento El Niño de 1997-98. En efecto, la duración del estado larvario es variable y depende de las condiciones ambientales para pasar a la fase bentónica (cf. Lea \& Béarez 1999); eso, obviamente, dentro de ciertos límites, los cuales por lo general se desconocen. Blache (1977: 132) estima que la duración de la vida larvaria de Hildebrandia sp., del Atlántico tropical, es aproximadamente de 6 meses, lo que efectivamente permite un transporte por las corrientes oceánicas sobre distancias importantes. Si consideramos una velocidad de la corriente subsuperficial del orden de $20 \mathrm{~cm} / \mathrm{s}$ (Brockmann et al. 1980), la distancia de $2000 \mathrm{~km}$ podría ser recorrida en 4 meses. Cabe también mencionar aqui que, según Lecomte-Finiger (1992), las larvas leptocéfalas de Anguilla anguilla (Linnaeus 1758) no sólo estan transportadas en forma pasiva por las corrientes si no que probablemente nadan en forma activa, lo que acorta su viaje trans-atlántico.

Raju (1985) y Charter (1996) señalan que las larvas de congrios pueden ser muy numerosas, sobre todo en el mes de mayo y que los juveniles aparecen a partir del mes de enero. El fenómeno El Niño 1997-98, catalogado como fuerte, se inició en marzo de 1997 para fortalecerse en octubre-noviembre y terminar en mayo del 98 (Fig. 2).

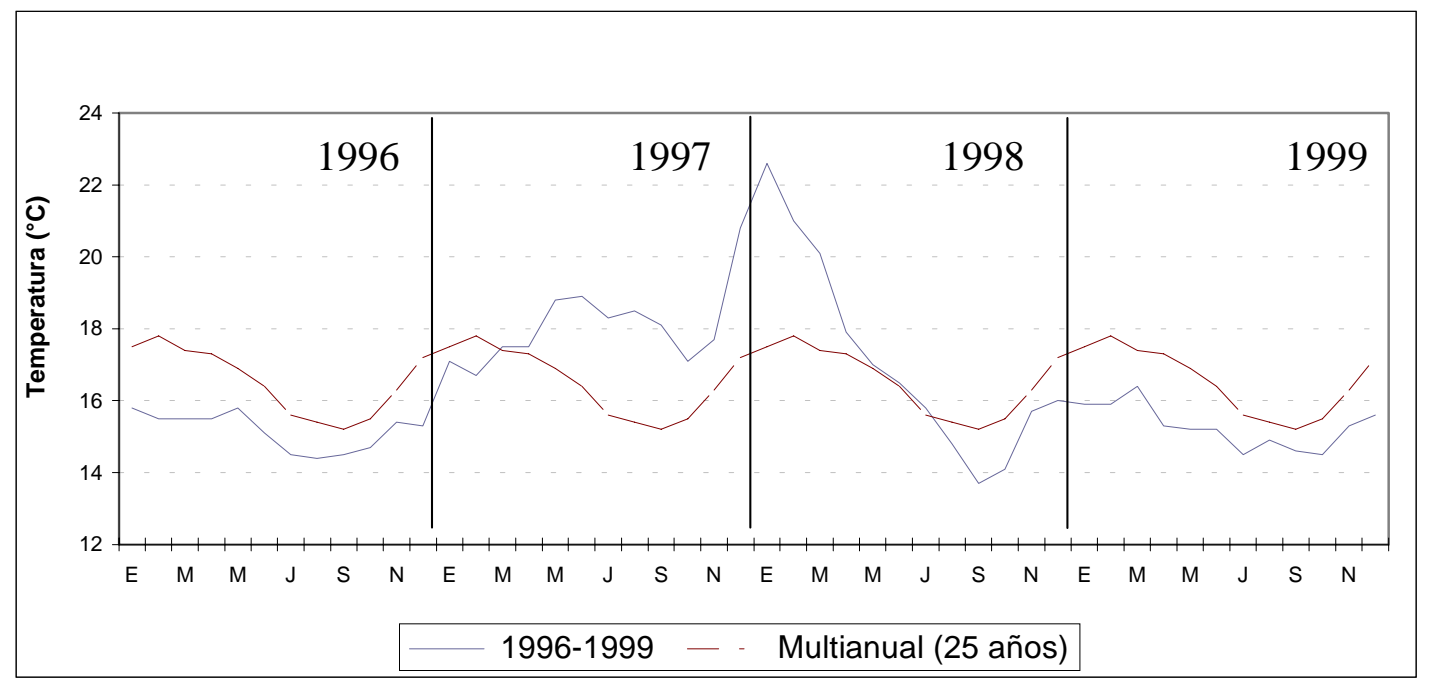

Figura 2

Variación de la temperatura superficial del mar en la estación costera fija de Ilo.

Variation of the sea surface temperature at the permanent coastal station of Ilo. 
El individuo estudiado podría muy bien haber sido transportado desde el noroeste por las aguas cálidas propagandose hacia el sur durante el evento ENSO. Aunque es difícil saber cual podría haber sido el mecanismo de transporte del pez, se sabe que durante un episodio El Niño se fortalece la corriente de Cromwell así como su rama sureña, la corriente subsuperficial peruanochilena (Brockmann et al. 1980, Lukas 1981, Icochea \& Rojas 2001). Conociendo las bajas capacidades de natación de los anguilliformes, es mas probable que el congrio haya viajado hasta Ilo en forma de larva, para llegar en forma de alevín y después haberse transformado en juvenil sedentario en el área, donde hubiera sobrevivido el tiempo suficiente como para ser capturado por un pescador local.

\section{Conclusión}

La ictiofauna peruana es muy diversa y se enriquece de los peces migrantes tanto del norte como del sur, en función de las fluctuaciones oceanográficas y climáticas, o sea del balance "corriente de Humboldt / fenómeno El Niño", lo que también se conoce ahora como "La Niña / El Niño". No es entonces sorprendente descubrir nuevos registros para el país después de un fuerte evento ENSO (El Niño-Southern Oscillation). Sin embargo, pensamos que el seguimiento del ictioplancton a lo largo de la costa peruana y sobre todo el estudio de la propagación de las especies tropicales hacia el sur durante un evento El Niño sería muy provechoso para nuestro conocimiento de los fenómenos de dispersión larvaria y colonización o recolonización de habitats por los peces.

\section{Agradecimientos}

Al Director del laboratorio costero del Imarpe-Ilo, Ing. Marco Quiróz Ruíz por las facilidades prestadas en la preservación y el estudio del especimen. A John McCosker y Juan Martín Díaz de Astarloa por haber aceptado leer el manuscrito y proveer sugerencias valiosas. A David Smith por facilitar las informaciones sobre los especimenes de $R$. nitens conservados en el USNM.

\section{Literatura Citada}

Béarez P. 1996. Lista de los peces marinos del Ecuador continental. Revista de Biología Tropical 44(2): 731-741.

Blache J. 1977. Leptocéphales des poissons anguilliformes dans la zone sud du Golfe de Guinée. Faune Tropicale 20: 1-381.
Böhlke EB (ed.). 1989. Fishes of the western North Atlantic. Part 9, Vol. 1. Orders Anguilliformes and Saccopharyngiformes, 655 p. Sears Foundation for Marine Research, New Haven.

Brockmann C, Fahrbach E, Huyer A \& L SMITH. 1980. The poleward undercurrent along the Peru coast: 5 to $15^{\circ}$ S. Deep-Sea Research 27A: 847-856.

Charter SR. 1996. Congridae: conger eels. En: Moser HG (ed.) The early stages of fishes in the California Current region. California Cooperative Oceanic Fisheries Investigations (CalCOFI) Atlas 33: 100-117.

Chirichigno N \& RM CORNEJO. 2001. Catálogo comentado de los peces marinos del Perú. 314 p. Publicación Especial del Instituto del Mar del Perú, Callao.

Chirichigno N \& J Vélez. 1998. Clave para identificar los peces marinos del Perú, 496 p. Publicación Especial del Instituto del Mar del Perú, Callao.

Icochea L \& E Rojas. 2001. La Corriente Cromwell y sus variaciones en los $0^{\circ} \mathrm{N}, 110^{\circ} \mathrm{W}$. En: Espino M, Samamé M \& C Castillo (eds), La merluza peruana (Merluccius gayi peruanus): biología y pesquería: 20-28. Instituto del Mar del Perú, Callao.

Jordan DS \& CH Bollman. 1890. Descriptions of new species of fishes collected at the Galápagos Islands and along the coast of the United States of Colombia, 1887-88. En: Scientific results of explorations by the U. S. Fish Commission steamer Albatross. Proceedings of the United States National Museum 12(770): 149-183.

Jordan DS \& BW Evermann. 1927. New genera and species of North American Fishes. Proceedings of the California Academy of Sciences 16(15): 501-507.

Lea RN \& P Béarez. 1999. Occurrence of Chilara taylori (Ophidiidae), an eastern North Pacific cusk-eel from Ecuadorian waters. Cybium 23(1): 99-100.

Lecomte-Finiger R. 1992. Growth history and age at recruitment of European glass eels (Anguilla anguilla) as revealed by otolith microstructure. Marine Biology 114(2): 205-210.

Lukas R. 1986. The termination of the Equatorial Undecurrent in the eastern Pacific. Progress in Oceanography 16: 63-90.

Massay S. 1983. Revisión de la lista de los peces marinos del Ecuador. Boletín Científico y Técnico, Instituto Nacional de Pesca, Guayaquil 6(1): 1-113.

Nelson JS. 1994. Fishes of the world, 600 p. John Wiley \& Sons, New York.

Raju SN. 1985. Congrid eels of the eastern Pacific and key to their leptocephali. U.S. Department of Commerce, NOAA Technical Report NMFS 22: 1-19. 
Smith DG. 1989. Family Congridae. En: Böhlke EB (ed.), Fishes of the western North Atlantic. Part 9, Vol. 1. Orders Anguilliformes and Saccopharyngiformes: 460-567. Sears Foundation for Marine Research, New Haven.

Smith DG. 1995. Congridae. Congrios. En: Fischer W, F Krupp, W Schneider, C Sommer, KE Carpenter \& V Niem (eds.), Guía FAO para la identificación de especies para los fines de la pesca. Pacífico centro-oriental. Vol. II: 1026-1035. FAO, Roma.
Swainson W. 1838. The natural history of fishes, amphibians, and reptiles, or monocardian animals. Vol. I. 368 p. Lardner's Cabinet Cyclopaedia. Longman, Orme, Brown, Green and Longmans, and John Taylor, London. 